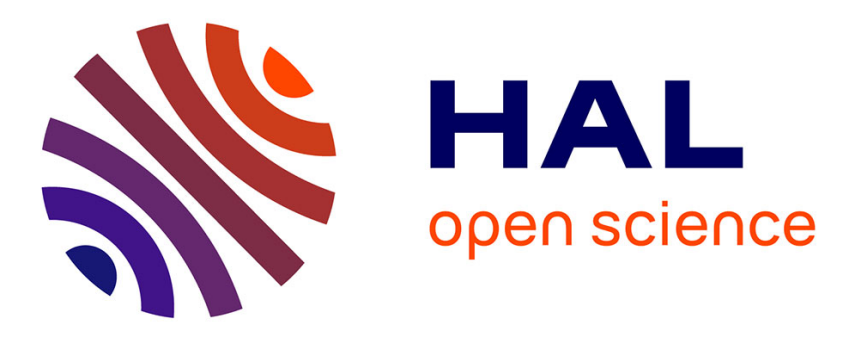

\title{
Control law synthesis for distributed multi-agent systems: Application to active clock distribution networks
}

Anton Korniienko, Gérard Scorletti, Eric Colinet, Eric Blanco, Jerome Juillard, Dimitri Galayko

\section{To cite this version:}

Anton Korniienko, Gérard Scorletti, Eric Colinet, Eric Blanco, Jerome Juillard, et al.. Control law synthesis for distributed multi-agent systems: Application to active clock distribution networks. 2011 American Control Conference - ACC 2011, IEEE, Jun 2011, San Francisco, CA, United States. pp.4691 - 4696, 10.1109/ACC.2011.5991295 . hal-01181176

\section{HAL Id: hal-01181176 https://hal.science/hal-01181176}

Submitted on 29 Jul 2015

HAL is a multi-disciplinary open access archive for the deposit and dissemination of scientific research documents, whether they are published or not. The documents may come from teaching and research institutions in France or abroad, or from public or private research centers.
L'archive ouverte pluridisciplinaire HAL, est destinée au dépôt et à la diffusion de documents scientifiques de niveau recherche, publiés ou non, émanant des établissements d'enseignement et de recherche français ou étrangers, des laboratoires publics ou privés. 


\title{
Control Law Synthesis for Distributed Multi-Agent Systems: Application to Active Clock Distribution Networks
}

\begin{abstract}
A. Korniienko, G. Scorletti, E. Colinet, E. Blanco, J. Juillard, D. Galayko
Abstract - In this paper, the problem of active clock distribution network synchronization is considered. The network is made of identical oscillators interconnected through a distributed array of phase-locked-loops (PLLs). The problem of the PLL network design is reformulated, from a control theory point of view, as a control law design for a distributed multi-agent system. Inspired by the decentralized control law design methodology using the dissipativity input-output approach, the particular topology of interconnected subsystems is exploited to solve the problem by applying a convex optimization approach involving simple Linear Matrix Inequality (LMI) constraints. After choosing the dissipativity properties which is satisfied by the interconnection matrix, the constraints are transformed into an $H_{\infty}$ norm constraint on a particular transfer function that must be fulfilled for global stability. Additional constraints on inputs and outputs are introduced in order to ensure the desired performance specifications during the $H_{\infty}$ control design procedure.
\end{abstract}

\section{INTRODUCTION}

$\mathrm{T}$ HE active clock distribution network (Fig.1) can be used as an alternative way to distribute the clock in synchronous many-core microprocessor systems. It has a large number of advantages in terms of perturbation rejection, robustness properties and power consumption [1-6]. In these systems, synchronization is crucial to ensure a correct system operation. Additionally, it is required that the generated clock signal is slightly sensitive to the external perturbations: temperature, noise and power, i.e. it possesses some signal purity properties. The network is generally composed of identical, independent voltage controlled oscillators (VCOs represented with dots in the right part of Fig. 1) that are spatially separated and connected in a two dimensional regular grid with phase detectors (PDs, represented with rectangles in Fig 1) which are located in between adjacent $V C O$ s in order to compensate the clock propagation delays; and identical filters (for sake of cleanness, represented by $F$ only in the left part of Fig 1). In general, each oscillator can be followed by a frequency divider ( $1 / d$ in the left part of Fig. 1) in order to distribute lower frequency clock signal throughout the network. The overall network is modelled as an interconnection of multiple input phase locked loop (PLL) nodes represented in the left part of Fig. 1 with $m_{i}$ shared phase detectors. In general, PLLs can be digital (All Digital PLLs) [4,7]. Depending on the application, one or several reference inputs can be introduced in the network. These reference oscillators must be synchronized and serve to enforce a common network clock frequency. Dynamically speaking, the described system has a much more complex behaviour than conventional clock distribution network. It is actually an automatic control system made of several feedback loops and caution must be taken to ensure stability of the overall system. Due to its complexity, classical microelectronics methods and tools fail to analyze and efficiently design this class of systems. In this paper, we illustrate how to apply control theory tools in order to ensure appropriate working operation of the whole system.

Methods for designing a stand-alone PLL are well-known in the field of microelectronics [8,9]. From a control system point of view, a PLL represents an LTI feedback dynamical system in the phase domain and one can design quite easily a controller ensuring good stability margins and performance requirements using standard control theory tools [8-11]. However, there is no guarantee for the global network that it convergences to the "synchronous state" even though each node is identical and properly designed to ensure the local convergence on an average input signal. There is no guarantee neither that the performance will not be degraded by the network. These aspects of global network inter-connection and nodes coupling are very important and must be taken into account during the system design procedure.

One of the most important results in understanding of global network dynamic or, more-generally, behavior of networked multi-agent systems is the work [12] where the authors give a necessary and sufficient stability condition for such LTI network. They use a transformation of the Laplacian interconnection matrix allowing the expression of the global stability test in the form of simultaneous stability tests of $N$ independent systems that have different feedback gains. Since these gains are defined by Laplacian eigenvalues (in general complex), one may apply in straight manner the Nyquist stability test of the global network. The paper [12] gave rise to a large number of various papers that use the same transformation to specify the stability test based on variety of approaches such as passivity, $\mathcal{L}_{2}$ gain [13-18], more general IQC characterization [19,20]. These papers deal with different set of additional problems such as nonlinear interconnections, effects of delay propagation and structure switching, but all of them are tools allowing only the stability analysis. However, there are two extensions to the design of a control law based on the result of [12].

A. Korniienko, G. Scorletti and E. Blanco are with Laboratoire Ampère, UMR CNRS 5005, Ecole Centrale de Lyon, 36 Av. Guy de Collongue 69134 Ecully cedex, France. anton.korniienko@ec-lyon.fr, gerard.scorletti@ec-lyon.fr.

A. Korniienko, E. Colinet are with CEA, LETI, MINATEC, 17 rue des martyrs, 38054 Grenoble Cedex 9, France, anton.korniienko@cea.fr, eric.colinet@cea.fr.

Jérome Juillard is with SSE, SUPELEC, Gif-sur-Yvette, France. jerome.juillard @ supelec.fr.

Dimitri Galayko is with UPMC Sorbonne Universités, LIP6 lab, 4, place Jussieu 75252 Paris Cedex 05, France. dimitri.galayko@lip6.fr. 
The first one is the robust control design pointed out in [12, 21, 22]. Using the Gershgorin theorem [23], authors propose to characterize the domain where all eigenvalues of the interconnection matrix must lie. Thereafter, they propose to express the feedback gain resulting from the transformation in the form of an uncertainty characterized by a certain norm. Further, one can apply a local robust control design guarantying the stability for all possible complex uncertainties using such a norm description and as a consequence ensuring the stability for all associated interconnection topologies. This proposed approach may be a suitable solution for the design problem. Nevertheless, the norm constraint that has to be guaranteed for the global stability is extremely conservative. It is actually based on a norm constraint on the complementary sensivity function $T$ that should have a maximum transmission gain not greater than one. As it is well-known [24], the maximum value of the complementary sensivity function magnitude is bigger or equal to 1 . So far, there is no successful implementation in the literature of such approach guaranteeing stability and desired performance in the same time for a multi-agent system.

The second extension is based on the simultaneous stabilisation of the $N$ independent systems according to the Theorem 3 in [12]. The authors of [25] use the static state feedback control law as a solution of an LMI optimisation problem. It is only possible in the case when the relative states are available for the control. Otherwise, the observed based solution is used in [26-28]. However there is no efficient method proposed to compute the control law that ensures stability as well as performance criteria besides a convergence rate to the steady-state (synchronous or consensus state). Moreover, for the control law of [26-28], a communication network able to transmit arbitrary information (controller and observer states) is required.

In the present paper, we propose a new approach that ensures the overall system stability as well as performance criteria. Additionally, the proposed control law requires only a local system output relative exchange (relative output errors) and is the solution of a convex LMI optimisation problem.

It turns out that in the literature of decentralized control, the problem of control law design for interconnected subsystems has been deeply treated. The authors of [29] solve this issue using the input-output approach. They use a dissipativity characterization [30] of subsystems which is more general than the passivity or $L_{2}$ gain as in [13-18]. They solve afterwards LMI conditions of local controllers existence that ensure the global stability and performance. However, since the subsystems that were considered in [29] are not necessary identical, the complexity of the condition they use depends on the overall number of network nodes. This potentially leads to some computational problem difficulties because a set of LMI conditions has to be ensured for every single node. On the other hand in practice, depending on the problem, the subsystems to control are very often identical at least with the same structure, as in our application of PLLs synchronization. Reasonably speaking, this property should be exploited to release some constraints on the controller design procedure.

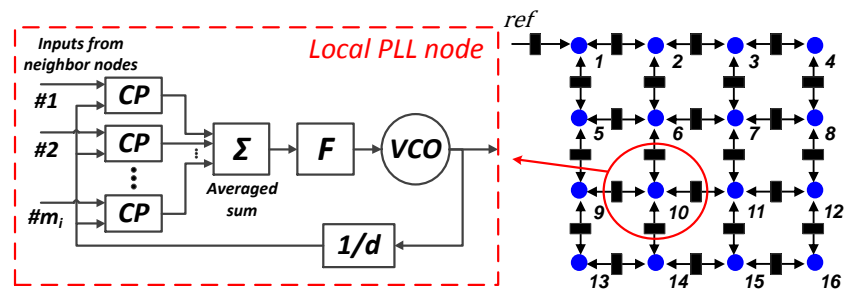

Fig. 1. Active clock distribution network

In the present paper inspired by the decentralized control in [29], we propose a design method that exploits the fact that nodes distributed in the network are identical. To do so, the dissipativity condition guaranteeing the overall network stability is transformed to an $H_{\infty}$ constraint on a transfer function involving only one node dynamic. It significantly reduces the computational complexity of the filter design algorithm. By applying an $H_{\infty}$ controller design ensuring this constraint to one single node, one can thus guarantee the stability of the overall network with specified condition on the interconnection matrix. Furthermore, we make sure that the performance constraints are locally fulfilled by the controller and we test afterwards possible performance degradation due to the interconnection.

The paper is organized as follows: the second section gives the used notations and preliminaries, in the third section we describe the general problem formulation. The main result is presented in the fourth section of the paper and its numerical application in fifth. We conclude the paper by the conclusion and the perspectives.

\section{NOTATION AND PRELIMINARIES}

Definition 1: A stable LTI causal operator $T$ with input $r$ and output $\varphi$ is strict $\{X, Y, Z\}$-dissipative, if there exist a real $\varepsilon>0$, real matrices $X=X^{T} \leq 0, Y, Z=Z^{T} \geq 0$ such that, $\left[\begin{array}{cc}X & Y \\ Y^{T} & Z\end{array}\right]$ is a full rank matrix and with $\varphi=T(r)$ :

$$
\begin{aligned}
& \int_{0}^{+\infty}\left[\begin{array}{l}
r(t) \\
\varphi(t)
\end{array}\right]^{T}\left[\begin{array}{cc}
X & Y \\
Y^{T} & Z
\end{array}\right]\left[\begin{array}{l}
r(t) \\
\varphi(t)
\end{array}\right] d t \leq-\varepsilon I \\
\Leftrightarrow & {\left[\begin{array}{c}
I \\
T(j \omega)
\end{array}\right]^{*}\left[\begin{array}{cc}
X & Y \\
Y^{T} & Z
\end{array}\right]\left[\begin{array}{c}
I \\
T(j \omega)
\end{array}\right] \leq-\varepsilon I, \forall \omega \in R }
\end{aligned}
$$

If the inequality (1) is satisfied with $\varepsilon=0$, the operator is thus called $\{X, Y, Z\}$-dissipative.

$\star$ denotes Lower Fractional Transformation (LFT), for $H=\left[\begin{array}{ll}H_{11} & H_{12} \\ H_{21} & H_{22}\end{array}\right]$ it is defined as: 


$$
(H \star G)(s)=H_{11}(s)+H_{12}(s) G(s)\left(I-H_{22}(s) G(s)\right)^{-1} H_{21}(s)
$$

$\otimes$ denotes the Kronecker product of two matrices defined by $A \otimes B=\left[a_{i j} B\right]$.

The $H_{\infty}$ norm of a stable LTI system $T$ is the maximal singular value of its transfer function that is formally defined as: $\|T\|_{\infty}=\sup _{\omega \in \mathbb{R}^{+}} \bar{\sigma}(T(j \omega))$.

\section{PROBLEM STATEMENT}

Let us consider the network of Fig. 1 where each node should be synchronized with its neighbours. The problem that has to be solved can be expressed as "find a control law $F$ that achieves the synchronization of the overall clock distribution network in a specified time for some fixed inter-connection structure and some specified clock signal purity”.

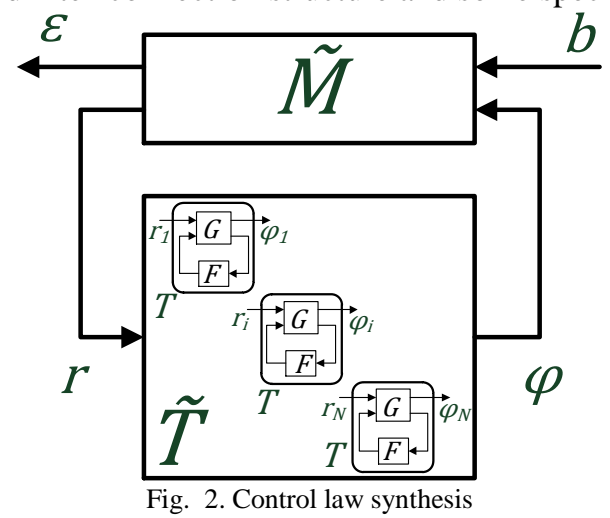

To design the controller of a stand-alone PLL, one usually models it in the phase domain [8-10]. A periodic signal is represented by its phase growing as a ramp with certain speed corresponding to the instantaneous oscillator frequency. Then the phase domain model is linearized around its operation point. Supposing that PLL will work close to this trim point, the controller can be efficiently designed. The same procedure has to be performed in the context of coupled PLLs network too. This allows to have not only a valid LTI model of the network but also to do not take into account some types of issues such as an auto-sampling in the case of digital PLLs and mode-locking state which can be treated separately as in [31, 32] and $[1,2,4]$.

The phase domain LTI model of the overall network is thus expressed as (2) and Fig.2.

$$
\left\{\begin{array}{c}
{\left[\begin{array}{c}
\varepsilon(t) \\
r(t)
\end{array}\right]=\left[\begin{array}{ll}
M_{11} & M_{12} \\
M_{21} & M_{22}
\end{array}\right]\left[\begin{array}{c}
r e f(t) \\
\varphi(t)
\end{array}\right]=\widetilde{M}\left[\begin{array}{c}
r e f(t) \\
\varphi(t)
\end{array}\right]} \\
\tilde{T}=I_{N} \otimes T=I_{N} \otimes(G \star F)
\end{array}\right.
$$

with $\varphi_{i}(t), r_{i}(t) \in \mathbb{R}^{p}, \forall i=1 . . N$

In Fig. 2, $G$ represents the part of a node that has to be controlled, $F$ the controller to be designed, $\widetilde{T}$ is the diagonal LTI matrix consisting of the $N$ identical LTI models $T$ and the block $\widetilde{M}$ is the interconnection matrix. Although in our application the subsystem $T$ (elementary PLL node) is a single-input single-output (SISO) system, in order to obtain a result as general as possible, we cover MIMO case with blocks $T$ of dimension $p \times p$. The vector $r$ is the vector of input mean values of the nodes, $\varphi$ is the vector of its outputs i.e. its local phase values, $b$ is the vector of external inputs and $\varepsilon$ is the vector of errors to be minimized depending on the performance specification. Indeed, the performance requirements can be expressed by the minimization of the $H_{\infty}$ norm for the transfer function between $b$ and $\varepsilon$. The mathematical expression of the performance objectives allows us not only to use the weighting transfer function approach for more precise frequency domain specifications, but also to generalize the problem to any problems of general networked multi-agent systems. Indeed, all engineering tasks such as: consensus, coordination, cooperation of subsystems can be expressed mathematically as an $H_{\infty}$ norm less than a given level $\gamma$ between some performance inputs and outputs [24] provided that the overall system is stable. In the following section we focus on this more general mathematical problem:

\section{General problem formulation:}

Given an LTI system $G$, an interconnection matrix $\widetilde{M}$, find an LTI system $F$ such that:

1) Each subsystem $T$ separately as well as the overall network formation (2) is stabilized;

2) A given performance specification by restricting the $H_{\infty}$ norm of local transfer function between performance inputs and outputs is ensured.

\section{MAIN RESULT}

The described problem will be solved in two steps. First, a control law is designed such it ensures: a) the stability of each linear subsystem $T$ and the overall system; b) an $H_{\infty}$ norm less than a given level $\gamma$ between some performance input and some performance output of the subsystem $T$. This level of performance is guaranteed only for an individual node i.e. the 
"local" performance. As a consequence, the second step consists in testing the "global" performance (performance of a node relative to the overall network) degradation by the network interconnection.

Although the PLLs can have both digital and continuous elements (as in our application case), in this paper all digital blocks of each node are transformed into continuous time blocks by Tustin transformation so that the synthesis and analysis are performed in the continuous time domain. After the synthesis of the control law, an inverse continuous to digital time Tustin transformation necessary for the implementation is performed.

\section{A. Stability}

First, the problem of local and global stability is addressed. To do so, we focus on the loop represented in Fig.2 and (2) without any external inputs and outputs $(\varepsilon, b)$ i.e. $M_{11}=0, M_{12}=0, M_{21}=0, M_{22}=M$.

The main result of the paper can be summarized by the following theorem:

Theorem 1: Given the LTI system (2) and $p \times p$ real matrices $X=X^{T} \leq 0, Y, Z=Z^{T} \geq 0$, If

1) there exists positive definite matrix $Q \in \mathbb{R}^{N \times N}$ such that the interconnection matrix $M$ is $\{Q \otimes X, Q \otimes Y$, $Q \otimes Z\}$-dissipative

2)

with $\widehat{T}(j \omega)=(-X)^{1 / 2}\left(T+X^{-1} Y\right)\left(Z-Y^{T} X^{-1} Y\right)^{-1 / 2}$

$$
\|\hat{T}(j \omega)\|_{\infty}<1
$$

Then the overall system is stable.

Sketch of the proof (See [33] for the complete proof).

The proof is based on a graph separation argument [30,34]. It is possible to prove the global stability of a feedback of two subsystems if they ensure two complementary input-output quadratic constraints.

The first constraint is ensured by the dissipativity condition 1) on the interconnection matrix $M$ i.e. $M$ is $\{Q \otimes X, Q \otimes Y$, $Q \otimes Z\}$-dissipative.

The second constraint is actually ensured by the $H_{\infty}$ norm constraint (3). Indeed, it can be proved that (3) implies the strict $\left\{-Q \otimes Z,-Q \otimes Y^{T},-Q \otimes X\right\}$-dissipativity of the global diagonal operator $\tilde{T}$. It completes the proof.

Remark 1: The dissipativity condition on the interconnection matrix $M$ is an LMI condition for some given $X=X^{T} \leq 0$, $Y, Z=Z^{T} \geq 0$ :

$$
\left\{\begin{array}{c}
\exists Q \in \mathbb{R}^{N \times N}: Q=Q^{T}>0 \\
{\left[\begin{array}{cc}
I_{p N} \\
M
\end{array}\right]^{T}\left[\begin{array}{cc}
Q \otimes X & Q \otimes Y \\
Q \otimes Y^{T} & Q \otimes Z
\end{array}\right]\left[\begin{array}{c}
I_{p N} \\
M
\end{array}\right] \leq 0}
\end{array}\right.
$$

As it is known, the feasibility problem of an LMI condition can be tested by applying the convex optimization algorithm $[35,36]$ which can be efficiently solved using e.g. Matlab LMI Toolbox. It is possible to transform (4) into the form of a quasi-convex optimization problem the solution of which allows to choose the $X, Y, Z$ dissipativity characterization matrices. Further details can be found can be found in [33].

Remark 2: The Theorem 1 constraint (3) can be ensured by $H_{\infty}$ control law design see Fig.3. However, we exploit all possibilities of $H_{\infty}$ design as MIMO design tools [24] by adding the desired performance inputs and outputs and by applying the design procedure for the extended system.

It should be mentioned that the stability problem reduces to solving the feasibility problem of two LMI conditions: the condition on the interconnection matrix $M(4)$ and the condition on the $H_{\infty}$ norm of the local transfer function (3). In the case when the state feedback control is possible, i.e. the controller has access to all plant states, the overall order of the LMI condition is equal to $3 N+2(n+\mathrm{p})$. $n$ is the order of the subsystem $G$. The corresponding order of the simultaneous stabilization problem proposed in [25] is equal to $2 N n-n$. For a large number of multi-agents $N \geq 10$ with a plant order $n \geq 2$, the proposed stabilisation optimisation problem order is much smaller. Furthermore, it can solve the problems where the state feedback control is not possible.

\section{B. Performance}

The next step is the control law design ensuring some performance properties besides the stability constraints. For clarity of representation, the SISO case of node transfer function T, i.e. $p=1$, is only considered. The equivalent approach can be applied to more general MIMO cases.

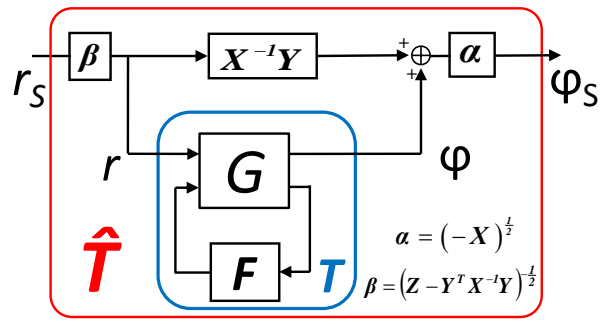


TABLE I

PERFORMANCE SPECIFICATIONS

\begin{tabular}{lcc}
\hline \hline \multicolumn{1}{c}{$\begin{array}{c}\text { Performance } \\
\text { objective }\end{array}$} & $\begin{array}{c}\text { Sensivity function to be } \\
\text { constrained }\end{array}$ & $\begin{array}{c}\text { Corresponding } \\
\text { weighted function }\end{array}$ \\
\hline $\begin{array}{l}\text { Perfect reference } \\
\text { (ramp) tracking }\end{array}$ & $S(s)=(1+G(s) F(s))^{-1}$ & $\left(W_{\varepsilon}(s) W_{r}(s)\right)^{-1}$ \\
Fixed response time & $S(s)$ & $\left(W_{\varepsilon}(s) W_{r}(s)\right)^{-1}$ \\
$\begin{array}{l}\text { VCO input, output } \\
\text { perturbations (noise, } \\
\text { temperature) rejection }\end{array}$ & $S S(s)=G(s)(1+G(s) F(s))^{-1}$ & $\left(W_{\varepsilon}(s) W_{r}(s)\right)^{-1}$ \\
& $F S(s)=F(s)(1+G(s) F(s))^{-1}$ & $\left(W_{\varepsilon}(s) W_{b}(s)\right)^{-1}$ \\
\hline
\end{tabular}

Fig. 3. Transformed model ensuring the global stability

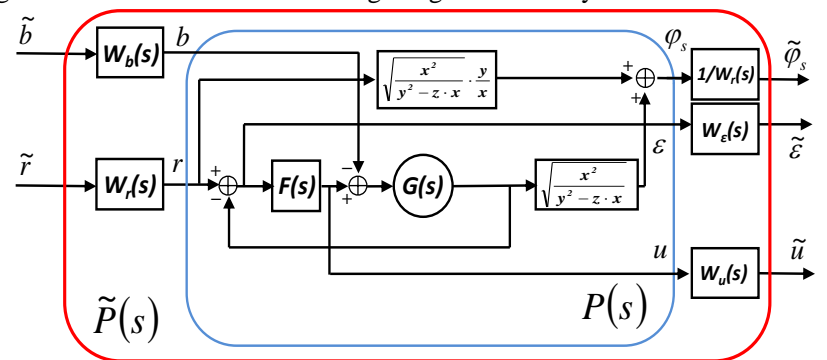

Fig. 4. Extended system with weighted functions fixing desired performance

Usual performance specification from the microelectronic point of view and the control system point of view with corresponding sensivity and weighting functions are presented in Table 1. By introducing additional performance inputs and outputs and related weighting functions to the scheme in Fig.3 the extended system description is deduced as in Fig.4.

The extended system in Fig. 4 can be expressed as:

$$
\tilde{P}(s)=\left[\begin{array}{cc}
W_{\varepsilon}(s) S(s) W_{r}(s) & -W_{\varepsilon}(s) G S(s) W_{b}(s) \\
W_{u}(s) F S(s) W_{r}(s) & -W_{u}(s) T(s) W_{b}(s) \\
W_{r}^{-1}(s) \hat{T}(s) W_{r}(s) & -W_{r}^{-1}(s) T_{r b}(s) W_{b}(s)
\end{array}\right]
$$

with weighting functions $W_{\varepsilon}(s), W_{u}(s), W_{r}(s), W_{b}(s)$ as a mathematical description of constraints with respect to the application.

The next step is the application of the $H_{\infty}$ design giving us the controller that ensures the stability of the feedback system $T$ (local stability of a node) and an $H_{\infty}$ norm of the transfer function $\tilde{P}(s)$ less or equal to a given level $\gamma$. If in addition $\gamma<1$ one thus obtains:

$$
\left\{\begin{aligned}
|S(j \omega)| & <\left|W_{\varepsilon}^{-1}(j \omega) W_{r}^{-1}(j \omega)\right| \\
|G S(j \omega)| & <\left|W_{\varepsilon}^{-1}(j \omega) W_{b}^{-1}(j \omega)\right| \\
|F S(j \omega)| & <\left|W_{u}^{-1}(j \omega) W_{r}^{-1}(j \omega)\right|, \forall \omega \in \mathbb{R} \\
|T(j \omega)| & <\left|W_{u}^{-1}(j \omega) W_{b}^{-1}(j \omega)\right| \\
& \|\hat{T}(s)\|_{\infty}<1
\end{aligned}\right.
$$

The algorithm of control law design can now be summarized as follows:

\section{Algorithm of the filter design:}

1) Choose the dissipativity characterization i.e. $X, Y, Z$ and apply the LMI optimization algorithm to find a matrix $Q$ that satisfies (4);

2) Select the weighting functions depending on the performance specifications;

3) Apply the $H_{\infty}$ control design to the extended system (5) of Fig.4;

4) If a controller achieving $\gamma<1$ is obtained then the local stability is guaranteed; the four first constraints in (6) ensure the local performance while the last one ensures the constraint (3) and thus by applying the Theorem 1 the global stability of the network.

5) If no controller is found or if the corresponding value of $\gamma$ is high such that the constraint (3) is not satisfied, reduce the weighing function constraints i.e. performance requirements. And then go to the step 3.

6) By computing the global transfer function from performance input $b$ to the performance output $\varepsilon$ test how the global performance is degraded compared to the local performance ensured by (6).

\section{NUMERICAL EXAMPLE}

For our application, a $4 \times 4$ Cartesian two dimensional ADPLL network is considered where the PLL at the upper-left corner has one additional external reference input as illustrated on Fig.1. The distribution network generates the clock at $0.97 \mathrm{GHz}$ with a frequency dividing factor of $d=4$. The total number of nodes (PLLs) is $N=16$. The VCO is modelled as a pure integrator $G(s)=K_{v c o} / s$ with gain $K_{v c o}=1.25 \cdot 10^{6} \mathrm{~Hz} /$ command units. The phase detectors are modelled by an adder followed by a gain $K_{p d}=20.5$ error units $/ \mathrm{rad}$. The real $m_{i}$ is the number of $i^{\text {th }}$ PLL inputs. All nodes in the network are assumed to be identical.

The linearized model in Fig. 1 can be transformed into the form of Fig. 2 described by (2) where $M_{22}$ is the $16 \times 16$ normalized adjacency interconnection graph matrix [12] where $i, j^{\text {th }}$ element is equal to $1 / m_{i}$ if $i^{\text {th }}$ node receives the information from $j^{t h}$ node and to 0 otherwise. The reference input is taken into account by $m_{i}$ as an additional input i.e. for the first node $m_{1}=3$.

In addition the reference $r e f(t)$, at the first node input with coefficient $1 / m_{1}$, and first error output $\varepsilon(t)=r e f(t)-$ $\varphi_{1}(t)$, are introduced in order to enforce the reference tracking. This results on the following interconnection matrix bloc definition: $M_{11}=1, M_{12}=\left[\begin{array}{llll}-1 & 0 & \cdots & 0\end{array}\right]$ and $M_{21}=\left[\begin{array}{cccc}1 / 3 & 0 & \cdots & 0\end{array}\right]^{T}$ and the solution of the algorithm described in the previous section: 
1. For $=-1.2, y=0.12, z=1$, we find $Q$ satisfying (4)

2. Based on the performance specification, the weighting functions are chosen (see dotted red line in Fig.5)

3. Applying the $H_{\infty}$ control design to the extended system (5) we obtain the performance level $\gamma=0.99$ and PI controller: $F(s)=\frac{2.92\left(s+1.44 \cdot 10^{5}\right)}{s}$

The corresponding sensitivity functions for the local PLL node as well as the performance for the transfer function of the first node in the overall PLL network are illustrated in Fig.5. As it can be seen the designed controller ensures:

1) The stability of the overall network:

- $\widehat{T}(s)$ satisfies the condition (3) of Theorem 1;

2) The local performance see [24]:

- Synchronization i.e. the ramp tracking without velocity errors since $|S(j \omega)|$ has the positive slope $+40 \mathrm{~dB} / \mathrm{dec}$ in the low frequency range. It confirms and extends the result in [37] that the PI consensus algorithm is sufficient for the synchronization of the identical networked clocks without any symmetric assumption on the network topology.

- Rejection of VCO input perturbations in all frequency range are at least $-35 d B$, see $|G S(j \omega)|$ transfer function in Fig.5. Furthermore, the $+20 \mathrm{~dB} / \mathrm{dec}$ slope in the low frequency range means that the system is able to reject the constant or slowly varying (drifting) VCO input perturbations. In other words, the non-identical central VCO frequencies (or clock speeds using the [37] terminology) which can be modeled as an input VCO perturbation, will not affect the final synchronization.

- Rejection of PD and VCO output perturbations in high frequencies are at least $-10 d B$, see $|T(j \omega)|$ transfer function in Fig.5.
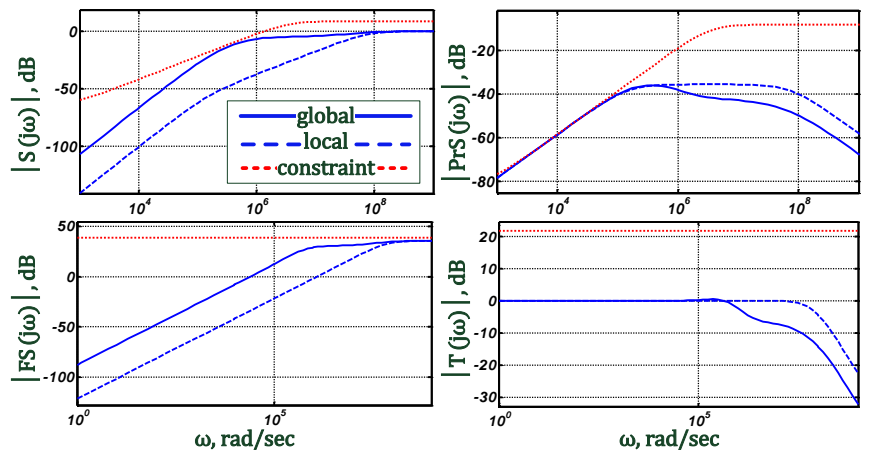

Fig. 5. Local sensivity functions (dashed blue line) of a PLL node, the corresponding global performance transfer function (full blue line) and the weighting functions (dotted red line).

\section{Global Performance discussion}

The algorithm of the control law design a priori ensures the local performance only i.e. for a single PLL node. Since the global system is an interconnection of such PLL nodes, the global performance (of a PLL node in the network) can be degraded (Fig. 5). Depending on the kind of interconnections, the difference in the performance level can be significant. Nevertheless, the global performance can still inherit some desired properties from the local case. An analysis of the topology impact which is based on the Mason's Direct Rule can be found in [38, 39]. One can actually show that with $\gamma<1$ the performance constraints are respected by some global transfer functions that take into account the network interconnection [33]. As it can be seen, the controller still ensures the synchronization without velocity error but with higher convergence time.

Finally, the bilinear continuous to discrete time transformation is performed to obtain the equivalent discrete control law. The proposed model herein is the real VLSI implementation of the network that takes into account the saturation of the phase detector and the filter outputs, the binary representation of the filter coefficients as well as the quantization of PD and DCO.

The synchronization efficiency with an external reference as in Fig.5 for a given randomly chosen initial state and ce-ntral frequencies $( \pm 10 \%)$ is illustrated in Fig.7. The PD out-put saturation is observed for large error value $( \pm 15)$. The sudden switches for tiny error values $( \pm 1)$ is caused by the nature of the used bang-bang PD (for more details see [4]). 


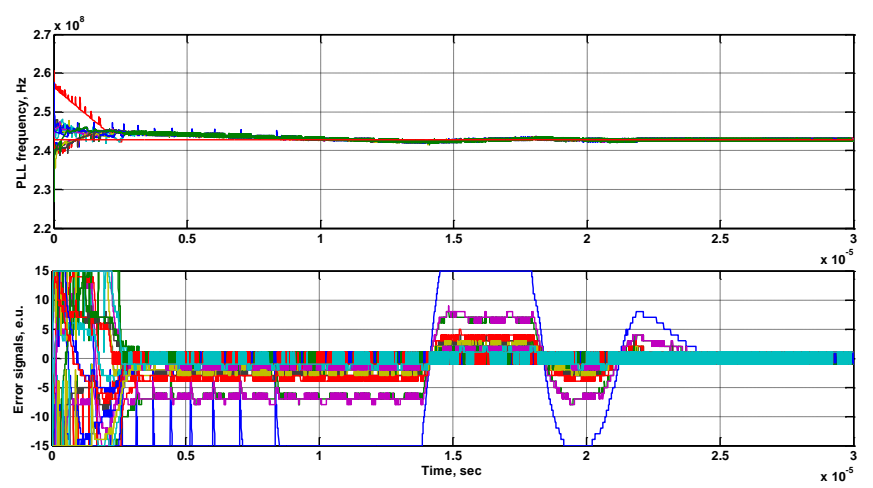

Fig. 6. Simulation results of the $4 \times 4$ ADPLL network, upper figure represents the evolution of node frequencies, bottom - the evolution of its relative phase error signal. Each PLL node is presented with a unique color.

\section{CONCLUSION}

In this paper we have proposed a method of control law design for an LTI networked multi-agent system ensuring besides the local agent stability, the stability of the overall network as well as the local performance requirements. Although the global performance is degraded by the network interconnection, the shape of corresponding transfer functions is conserved ensuring the synchronization of the network. The next step in this work should introduce the global performance objectives during the design procedure. Another perspective is to examine through the $\mu$-analysis tools the influence of uncertainties on the overall system stability and performance. The perturbations, unavoidable in practice, can include the models nonlinearities, delays or possible dispersions. This study should allow the realization of the solid-state PLL network VLSI circuit implementation.

\section{ACKNOWLEDGMENT}

This work is supported by the French National Agency of Research (ANR) through the HODISS project.

\section{REFERENCES}

[1] G. A. Pratt, and J. Nguyen, "Distributed Synchronous Clocking", IEEE Trans. Parallel and Distributed System, vol. 6, no. 3, pp. 314-328, March 1995.

[2] V. Gutnik, and A. P. Chandrakasan, "Active GHz Clock Network Using Distributed PLLs", IEEE Journal of Solid-State Circuits, vol. 35, no. 11, pp. $1553-1560$, Nov. 2000.

[3] A. Korniienko, E. Colinet, G. Scorletti, and E. Blanco, " $\mathrm{H}_{\infty}$ loop shaping control for distributed PLL network", IEEE Ph.D. Research in Microelectronics \& Electronics PRIME-2009, Cork, Jul., pp. 336-339.

[4] A. Korniienko, E. Colinet, G. Scorletti, E. Blanco, D. Galayko, and J. Juillard, "A clock network of distributed ADPLLs using an asymmetric comparison strategy." Proc. 2010 IEEE Int. Symp. Circuits and Systems (ISCAS), May-June, Paris, pp.3212-3215.

[5] F. Anceau, "Une technique de réduction de la puissance dissipée par l'horlogerie des circuits complexes rapides", 4ème journées francoph. d'étude Faible Tension Faible Consommation (FTFC'2003), Paris, May.

[6] M. Saint-Laurent, and M. Swaminathan, "A multi-PLL clock distribution architecture for gigascale integration", IEEE Computer Society Workshop VLSI, May 2001. pp. 30-35.

[7] J.A. Tierno, A.V. Rylyakov, and D. J. Friedman "A Wide Power Supply Range, Wide Tuning Range, All Static CMOS All Digital PLL in 65 nm SOI", IEEE J. Solid-State Circuits, vol.43, no. 1, pp. 42-51, Jan. 2008.

[8] U.L. Rohde, Microwave and Wireless Synthesizers: Theory and Design., New York: John Wiley \& Sons, Inc., 1997.

[9] V. Kroupa, Phase Lock Loops and Frequency Synthesis, Chichester: John Wiley \& Sons, Inc., 2003.

[10] C. Kharrat, E. Colinet, and A. Voda, " $\mathrm{H}_{\infty}$ Loop shaping control for PLL-based mechanical resonance tracking in NEMS resonant mass sensors", Proc. 2008 IEEE Sensors, Lecce, Oct., pp.1135-1138.

[11] M. Houdebine, "Contribution pour l'amélioration de la robustesse et du bruit de phase des synthétiseurs de fréquences", Ph.D. dissertation, Nat. Polytech. Inst. Grenoble, 2007.

[12] J. Fax, and R. Murray, "Information flow and cooperative control of vehicle formations," IEEE Trans. Autom. Control, vol. 49, no. 9, pp. 1465 1476, Sep. 2004

[13] N. Chopra, and M. W. Spong, "On synchronization of networked passive systems with time delays and application to bilateral teleoperation."
Proc. SICE Annu. Conf., Okayama, Jan. 2005, pp. 3424-3429.

[14] N. Chopra, and M. W. Spong, "Output synchronization of nonlinear systems with time delay in communication.", Proc. 45th IEEE Conf. on Decision and Control, San Diego, Dec. 2006, pp. 4986-4992.

[15] N. Chopra, and M. W. Spong, "Delay-independent stability for interconnected nonlinear systems with finite L2 gain." Proc. 46th IEEE Conf. on Decision and Control. New Orleans, Dec. 2007, pp. 3847-3852.

[16] M. Arcak, "Passivity as a Design Tool for Group Coordination." IEEE Transactions on Automatic Control, vol.52, no.8, pp.1380-1390, Aug. 2007.

[17] H. Bai, M. Arcak, and J.T. Wen, "Adaptive design for reference velocity recovery in motion coordination.", Systems \& Control Letters, vol. 57, no. 8, pp. 602-610, Aug. 2008.

[18] H. Bai; M. Arcak, and J.T. Wen, "Group Coordination when the Reference Velocity is Available Only to the Leader: An Adaptive Design.", American Control Conference, New York, Jul. 2007, pp.5400-5405.

[19] U. Jonsson, C.-Y. Kao, H. Fujioka, "A Popov criterion for networked systems", Systems \& Control Letters, vol. 56, no. 9-10, pp. 603610, Sep.-Oct. 2007.

[20] C.-Y. Kao, U. Jonsson, H. Fujioka, "Characterization of robust stability of a class of interconnected systems", Automatica, vol. 45, no. 1, pp. 217-224, Jan. 2009

[21] R. Olfati-Saber, J. A. Fax, and R. M. Murray, "Consensus and cooperation in networked multi-agent systems.", Proc. IEEE, vol. 95, no. 1 , pp. 215-233, 2007.

[22] A.P. Popov, and H. Werner, "A Robust Control Approach to Formation Control", Proc. ECC 2009, Budapest, Aug.

[23] R. Horn and C. Johnson, Matrix Analysis, New York: Cambridge Univ. Press, 1985.

[24] S. Skogestad, I. Postlethwaite, Multivariable Feedback Control: Analysis and Design, 2nd ed., Chichester: John Wiley \& Sons, Ltd., 2005.

[25] P. Wieland, J.-S. Kim, H. Scheu, and F. Allgöwer, "On consensus in multi-agent systems with linear high-order agents.", Proc. 17th IFAC World Congress, Seoul, July 2008, pp. 1541-1546.

[26] P. Wieland, "From static to dynamic couplings in consensus and synchronization among identical and non-identical systems", Ph.D. dissertation, Inst. for Systems Theory and Automatic Control, Univ. 
Stuttgart, 2010.

[27] P. Wieland and F. Allgöwer, "On consensus among identical linear systems using input-decoupled functional observers", American Control Conference (ACC), pp. 1641-1646, July 2010.

[28] L. Scardovi, R. Sepulchre, "Synchronization in networks of identical linear systems", Automatica, vol. 45, no. 11, pp. 2557-2562, Nov. 2009.

[29] G. Scorletti, G. Duc, "An LMI approach to decentralized control", Int. J. Control, vol. 74, no. 3, pp. 211-224, 2001.

[30] P. J. Moylan and D. J. Hill, "Stability criteria for large-scale systems," IEEE Trans. Aut. Control, vol. AC-23, pp. 143-149, 1978.

[31] J.M. Akré, J. Juillard, D. Galayko, E. Colinet, "Synchronized State in Networks of Digital Phase-Locked Loops", 8th IEEE Inter. NEWCAS Conf., Montreal, June 2010, pp. 89-92.

[32] J.M. Akré, J. Juillard, S. Olaru, D. Galayko, E. Colinet, "Determination of the Behaviour of Self-Sampled Digital Phase-Locked Loops", 53rd IEEE Inter. Midwest Sympos. Circuits and Systems, Seattle, Aug. 2010, pp. 1089-1092.

[33] A. Korniienko, G. Scorletti, E. Colinet, "Control Law Design for Distributed Multi-Agent Systems", Ampere Res.Lab., Ecole Central de Lyon, Tech. Rap. 2011.

[34] A. Megretski, A. Rantzer, "System analysis via integral quadratic constraints.", IEEE Trans. Autom. Control, vol. 42, no. 6, pp 819-830, 1997.

[35] S. Boyd, L. E. Ghaoui, E. Feron, and V. Balakrishnan, Linear Matrix Inequalities in System and Control Theory, vol. 15 of Studies in Applied Mathematics, SIAM: Philadelphia, 1994.

[36] A. Ben-Tal, A. Nemirovski, Lectures on Modern Convex Optimization: Analysis, Algorithms, and Engineering Applications, MPSSIAM Series on Optimization, Philadelphia, 2001.

[37] R. Carli, A. Chiuso, L. Schenato and S. Zampieri. (2008). A PI Consensus Controller for Networked Clocks Synchronization. IFAC Proc.Volumes (IFAC-PapersOnline). Volume 17(1). Available: http://www.ifac-papersonline.net.

[38] S. Tonetti and R. Murray, "Limits on the network sensitivity function for homogeneous multi-agent systems on a graph," American Control Conference (ACC), Baltimore, Jul. 2010, pp. 3217-3222.

[39] S. Tonetti and R. Murray, "Limits on the network sensitivity function for multi-agent systems on a graph", California Institute of Technology, Pasadena, CA, Tech. Rep., 2009. 